\title{
Radiological imaging of rectal cancer
}

\author{
Lidija Lincender-Cvijetić1,2, Maja Banjin-Čardžić3 ${ }^{3}$ Sandra Vegar-Zubović ${ }^{4}$ \\ Dunja Vrcić 4
}

\author{
${ }^{1}$ Academy of Science and Arts \\ of Bosnia and Herzegovina, Sarajevo \\ Bosnia and Herzegovina \\ ${ }^{2}$ Faculty of Health Studies, University \\ of Sarajevo, Sarajevo, Bosnia and \\ Herzegovina \\ ${ }^{3}$ Clinics of Oncology, University Clinical \\ Center Sarajevo, Sarajevo, Bosnia and \\ Herzegovina \\ ${ }^{4}$ Clinics of Radiology, University Clinical \\ Center of Sarajevo, Sarajevo, Bosnia and \\ Herzegovina \\ Corresponding author: \\ Lidija Lincender-Cvijetić \\ Academy of Science and \\ Arts of Bosnia and Herzegovina \\ Bistrik 7 \\ 71000 Sarajevo \\ Bosnia and Herzegovina \\ lidija_lincender@hs-hkb.ba \\ Tel.: + 38761190121 \\ Fax.: + 38733560703
}

Received: 1 July 2011

Accepted: 11 June 2012

Copyright (c) 2012 by

Academy of Sciences and Arts of Bosnia and Herzegovina.

E-mail for permission to publish: amabih@anubih.ba

\begin{abstract}
This article discusses the possibilities of diagnosing abdominal imaging in patients with rectal cancer, detecting lesions and assessing the stage of the lesions, in order to select the appropriate therapy. Before the introduction of imaging technologies, the diagnosis of colorectal pathology was based on conventional methods of inspecting intestines with a barium enema, with either a single or double contrast barium enema. Following the development of endoscopic methods and the wide use of colonoscopy, colonoscopy became the method of choice for diagnosing colorectal diseases. The improvement of Computerized Tomography (CT) and Magnetic Resonance Imaging (MRI), gave us new possibilities for diagnosing colorectal cancer. For rectal cancer, trans-rectal US (TRUS) or endo-anal US (EAUS) have a significant role. For staging rectal cancer, the Multi Slice Computed Tomography (MSCT) is not the method of choice, but Magnetic Resonance Imaging (MRI) is preferred when it comes to monitoring the rectum. The role of the MRI in the T staging of rectal cancer is crucial in preoperative assessment of: thickness - the width of the tumor, the extramural invasion, the circumference of resection margin (CRM), and the assessment of the inclusion of mesorectal fascia. For successful execution of surgical techniques, good diagnostic imaging of the cancer is necessary in order to have a low level of recurrence. According to medical studies, the sensitivity of FDG-PET in diagnosing metastatic nodals is low, but for now it is not recommended in routine diagnosis of metastatic colorectal carcinoma.
\end{abstract}

Key words: Colorectal cancer, TRUS, MSCT, MRI, Staging.

\section{Introduction}

Colorectal cancer is a malignant disease on the rise. Annually, about 300,000 new cases of colorectal cancer are diagnosed per 500 million EU citizens. Colorectal cancer is the third most common cancer among men (only after lung and prostate cancer), and the second most common cancer among women (after breast cancer) (1). It represents the second cause of death in both sexes, in men after lung cancer and breast cancer in women. Colorectal cancer is the second most common cancer in general and the most common of the gastrointestinal tract cancers. It 
is equally common in both genders, mostly in those over 50 years of age. In about 50\% of cases the cancer occurs in the rectum and colon recto-sigmoid, the other $25 \%$ occurs in the sigma, while the remaining $25 \%$ is found in the rest of the colon. Rectal cancer is a major problem precisely because of its high incidence. For many years the primary radiological diagnostic method in diagnosing abnormalities of the colon and rectum was barium enema. Double contrast technique is used in detecting small lesions $(<1$ $\mathrm{cm}$ ), documenting inflammatory diseases and detecting rectal pathology. Before the introduction of colonoscopy, barium enema was the method of choice for screening colorectal cancer. The development of colonoscopy is changing the algorithm because, unlike colonoscopy, barium enema is a method of low sensitivity when it comes to detecting polyps and cancer (2) and it is performed only when colonoscopy is not possible or unsuccessful. With the use of Computerized Tomography (CT) imaging, Multi Slice Computed Tomography (MSCT), with the advantages of computerized colonography (CT colonography) and Magnetic Resonance Imaging (MRI), diagnosis of colorectal pathology, particularly the detection of the colon and rectal cancer, has been enhanced (3-6). These methods provide insight into intramural and extra-colic pathological changes in the colorectal region. Both of these methods have their limitations, particularly with older generation CT and MRI scans, in assessing the stage of the tumor (TU) in the area of the bowel wall and the invasion to regional lymph nodes. MSCT colongraphy remains the method of choice after the proven insufficiency of colonoscopy (7-11). In 2004, a 64-slice CT scan was introduced in the diagnostic process. Compared to the older CT generation, 64-slice CT scan has a shorter scanning time (7 sec for $40 \mathrm{~cm}$ ), with improved spatial and temporal resolution images. However, even in devices with the option of "low dose", there is still a high limit of radiation dose for colorectal screening (12).

Transrectal US (TRUS) is an accurate diagnostic method for rectal cancer compared to older CT and MRI technologies. MRI, with high resolution $\mathrm{T} 2$ weighted sequences, clearly depicts the details of the rectal wall and perirectal anatomy. The rectal wall can be recognized as three different layers: a thin inner line of low signal intensity represents the mucosal layer, a middle layer of high signal intensity represents the submucosa, and an outer layer of low signal intensity represents the muscularis propria $(13,14)$. The mesorectal fascia, which forms the boundary of the surgical excision plane in total mesorectal excision, is identified as a thin, low - signal intensity structure on the MRI, that envelops the rectum and the surrounding perirectal fat (14).

\section{Radiological imaging of rectal cancer}

Determining an optimal treatment plan for an individual patient with rectal cancer is a complex process. In addition to facing decisions regarding the intent of rectal cancer surgery, consideration must also be given to the validity of the treatment results, including the probability of maintaining or restoring normal bowel function/anal continence and preserving genitourinary functions. Careful patient selection with respect to particular treatment options and the use of sequenced multimodality therapy for selected patients, which combines chemo - radiation with operative treatment as part of the treatment regimen, is recommended. The main objective in diagnostically imaging patients with rectal cancer is to detect lesions and assess the extent of those lesions, in order to better assist the selection of appropriate therapy. Patients with rectal cancer appropriate for resection require complete staging evaluation, including full colonoscopy to evaluate the synchronous lesions, rigid 
proctoscopy to determine the location of the cancer, baseline computed tomography scans of the chest, abdomen and pelvis, and a complete physical examination.

The accessibility of evaluating rectal cancer by certain imaging modalities, such as endoanal US (EAUS) and MRI scan, makes preoperative assessments of the depth of tumor penetration and the presence of local lymph nodal metastases possible. Additional information regarding the extent of the disease and the occurrence of distant metastases can be determined preoperatively through CT scans. Thus, endorectal ultrasound, or endorectal or pelvic MRI, CT scans of the chest, abdomen and pelvis, are recommended for the preoperative staging of rectal cancer. A positron emission tomography scan is not routinely indicated at baseline in the absence of evidence of synchronous metastasis disease.

The most common method for determining the stage of colorectal cancer is the AJCC/TNM system CC VII (15). General $\mathrm{CT}$ and MSCT are now the most widely used methods in determining the stage of colorectal cancer (Figure 1 and Figure 2) $(9,10)$.

In contrast to some limitations of $\mathrm{CT}$ and MRI scans when it comes to predicting the

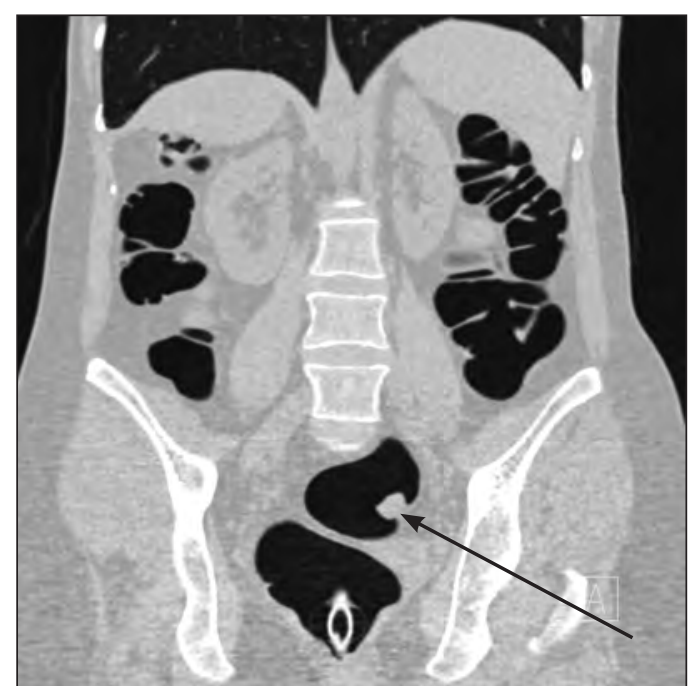

Figure 1 MSCT 2D - adenocarcinoma of recto-sigmoid colon. Black head arrows show the polypoid mass. stage of rectal cancer, high resolution MRI of the rectum has recently been proven to be highly accurate and reproducible in assessing the absolute extramural depth of tumor invasion in rectal cancer $(16,17)$. Phased array surface coil is preferred for high resolution MRI scanning of the rectum, because the endorectal coil MRI offers a limited field of vision, despite its superior spatial resolution: a complete assessment of perirectal structures is difficult with the endorectal coil because portions of the mesorectal fascia, mesorectal fat, and lymph nodes lie outside the field of view (16).

In the preoperative evaluation based on MRI scans, attention is given to an accurate assessment of the depth of tumor invasion, extra-mural invasion of the circumference of resection margin (CRM), and the mesorectal fascia, which is equal to the circumference border of the surgical excision (17-24). The standard surgical procedure for rectal resection includes the resection of the mesorectal fat and the associated lymph nodes. In patients with TU, extra-mural invasion radio, or chemo therapy is recommended before surgery, to reduce the risk of TU recurrence after surgery. The thickness of the colorectal wall due to TU, extra-tumor invasion, and the presence or absence of resec-

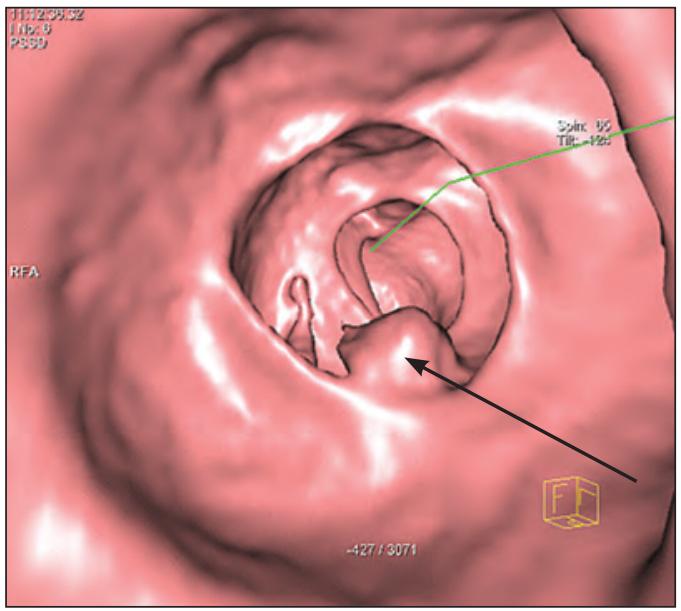

Figure 2 MSCT colonography - adenocarcinoma of rectum. Black head arrows show the polypoid mass. 
tion tumor circumferential surface are the main risks for relapse and further post-operative prognosis (17-24).

The identification and staging of rectal cancer by MRI scans is largely based on differences in T2 signal intensity between the tumor, the rectal wall and perirectal fat tissue. The tumor itself has an intermediate signal intensity which is between the high signal intensity of the submucosa, or perirectal fat tissue, and the low signal intensity of the muscular layer $(16,24)$. The absolute depth of extramural invasion of rectal cancer on MRI scans agrees well with pathological measurement, and the presence or absence of the tumor-effect in the CRM can be predicted accurately, with at least a 1 or 2 $\mathrm{mm}$ distance from which there is high risk of postoperative recurrence $(16,24,25)$. However, the exact depth of the extra-mural spread of TU is considered to have less importance, since all patients with T3 and T4, and possibly positive lymph nodes, receive preoperative chemo and radio therapy (9).

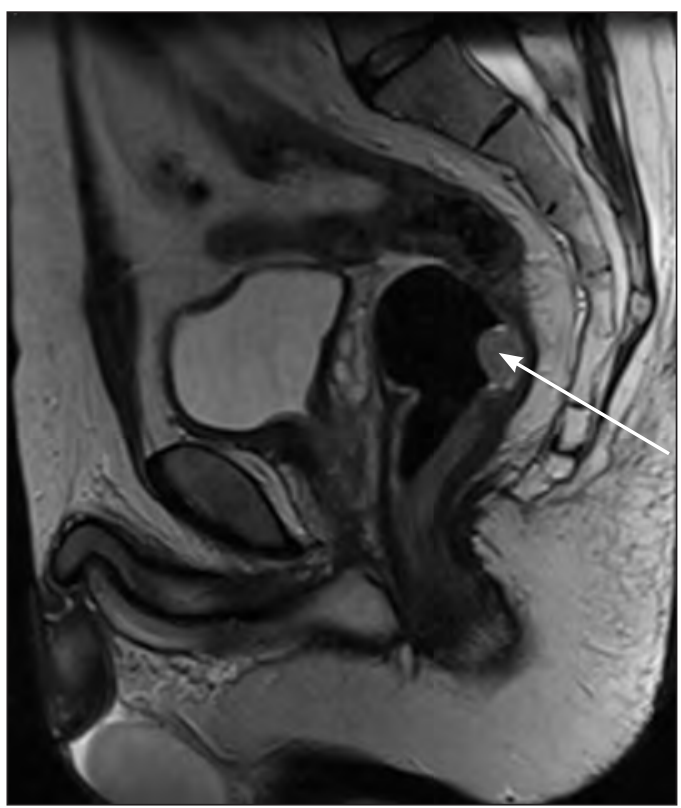

Figure $3 \mathrm{MRI}$ T2WI adencarcinoma of the posterior wall of the rectum. White head arrow shows the polypoid mass.
For successful diagnosis of colorectal carcinoma good bowel distention is crucial, when using either MSCT or MRI scans. In the case of poor distention during CT colonography it is possible to miss larger lesions $(9,25)$ if the colon is collapsed. In determining the stage of rectal cancer, colonic distension will improve lesion visibility but may alter the distance between the outer margin and mesorectal fascia, reassessing the extramural depth of tumor invasion (9). On MSCT or MRI images, rectal cancer appears

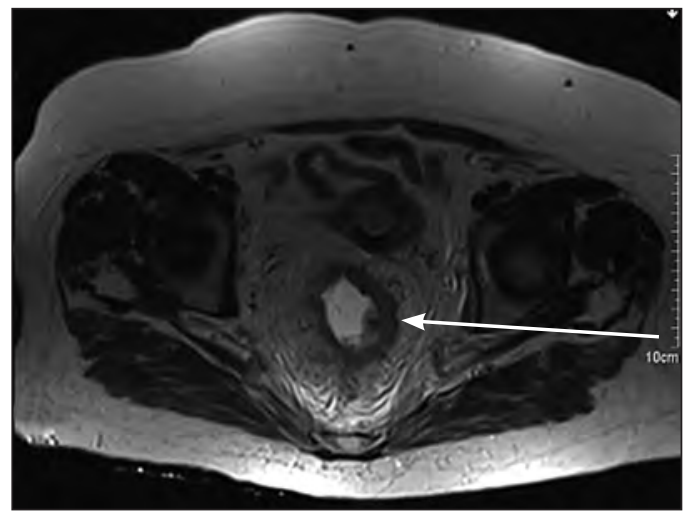

Figure $4 \mathrm{MRI}$ transverse cross-section of the rectum - adenocarcinoma. White head arrow shows asymmetrical wall thickening of rectum with an irregular surface.

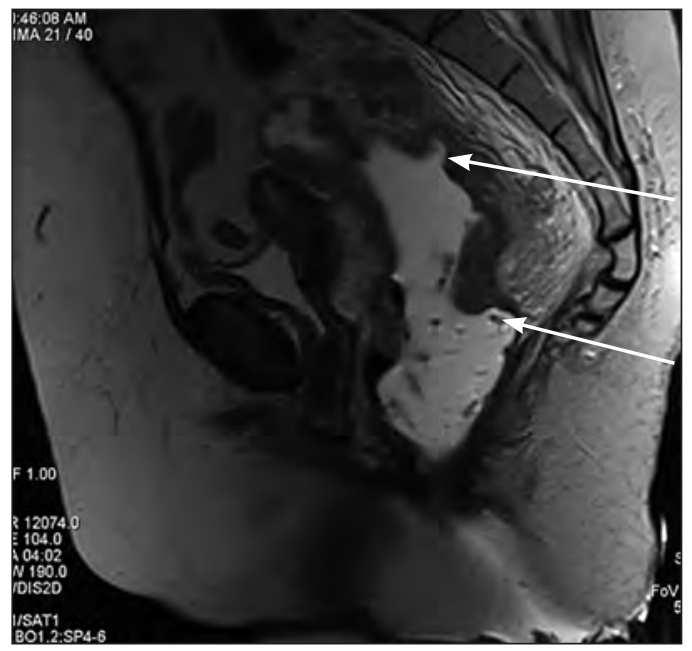

Figure $5 \mathrm{MRI}$ sagittal section of the rectum - adenocarcinoma. White head arrow shows asymmetrical wall thickening of rectum with an irregular surface. 
as discrete mass focal or a short-segmented wall thickening (Figure 3).

Asymmetrical wall thickening of rectum, with or without an irregular surface, suggests a neoplastic process (Figure 4, Figure 5) (26).

\section{Assessment of local tumor spread}

Diagnosis of tumor invasion beyond the bowel wall can be made with cross - section imaging, but only if the tumor mass extends directly into the surrounding muscle (levator anni, obturator internus, coccygeus, piriformis or gluteus maximus), or organs, obliterating the fat planes and enlarging the individual muscle, or enveloping the neighboring structures.

Accuracy of MSCT and MRI scanning in loco-regional staging has increased considerably thanks to advances in imaging technology in recent years. The diagnostic accuracy for polyps larger than one $\mathrm{cm}$ ranges from $60 \%$ to $100 \%$, while for cancer it is $100 \%$ (9).

Sensitivity of CT imaging in the evaluation of TU and T stage is $78 \%$ and $63 \%$ respectively, and specificity is $86 \%$ and $77 \%$ respectively. Sensitivity of the MRI scan in comparison with EAUS is $93 \%$ and $78 \%$ respectively (27). An MRI of the rectum, when it comes to assessing the extramural spread of the tumor, compared to CT scanning (which has its limitations), has greater diagnostic value (27-30).

The application of an endorectal coil has limitations in the width of field, despite its superior spatial resolution; analysis of the complete tumor expansion to the perirectal structure is insufficient because parts of the mesorectal fascia, fat, and mesorectal lymph nodes, outside the primary field, are not observed. It is similar with TRUS, which is more accurate than CT or MRI scans in evaluating the wall of the rectum and superficial rectal cancer. TRUS is limited in evaluating perirectal and mesorectal fascia due to limited tissue penetration $(26,28)$. T2 MRI sequences are most suitable for depicting the anatomy of the rectal wall and the spread of cancer in the perirectal space, as well as high-resolution T2 weight sequences imaging, with a non-breath-hold turbo spine echo sequences which was used in most studies $(13,23,24)$. Identifying and determining the stage of rectal cancer by MRI scan is based on the differences in T2 signal intensity between the tumor and the rectal wall, and the perirectal fat tissue. There is intermediate signal intensity between the high-signal intensity of the submucosa and perirectal fat, and low signal intensity of the muscular layer $(13,23)$.

Three dimensional radiation therapy to assess the borders of the tumor is based on MSCT imaging, which is not always the optimal method. The greatest restriction is the low contrast resolution. MRI imaging is more likely to determine smaller lesions, more accurately determining the volume of the tumor compared to CT scanning. The volume of the tumor detected on an MRI scan is smaller and shorter at the distal of the anal sphincter than the volume based on the CT scan. In planning radiotherapy, these results will result in a smaller volume of radiation, leading to dose reduction in the surrounding organs at risk (31).

Rectal cancer grows from mucosa and progressively spreads to deeper layers of the bowel wall. The outer margins of the rectum are smooth on CT and MRI imaging, and perirectal and pericolic adipose tissues have the same density on CT scans and the same signal intensity as on MRI. Spreading of the tumor outside the wall of the intestines is manifested as the irregular outer margin of the bowel wall with expansion to the soft tissue as well as to pericolic and perirectal adipose tissues (Figure 6). A similar expansion may occur in through the desmoplastic response of the peritumor tissues with inflammatory or congestive changes, resulting in a miscalculation of stage T2 and T3 lesions. 


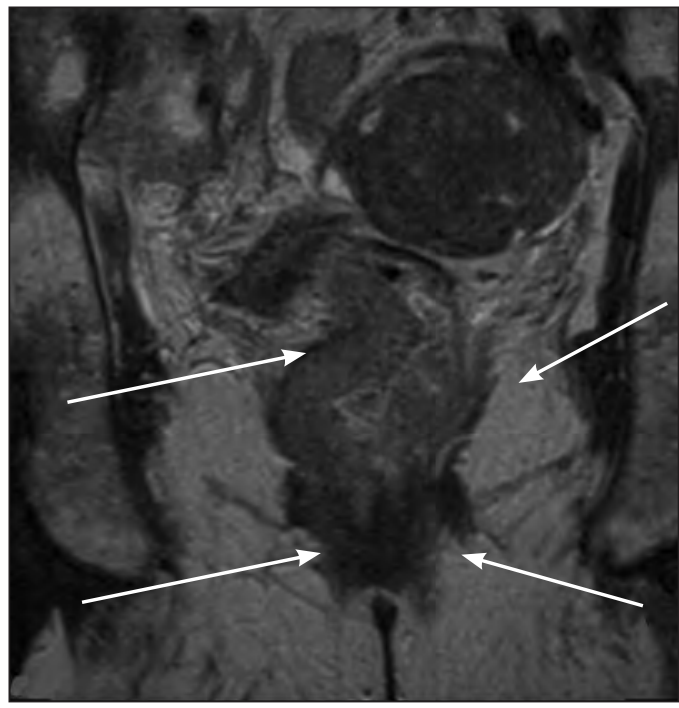

Figure $6 \mathrm{MRI}$ Ca rectum. White head arrows show the spread of the tumor outside the wall of the rectum.

Furthermore, a microscopic cancer invasion into the pericolic adipose tissue cannot be visualized on CT or MRI scans, making the T3 resemble T2 (9).

\section{Involvement of the lymph nodes in rectal cancer}

By following the anatomy of the lymphatic drainage of the colon, with present crosssectional imaging we can predict the spread of colorectal cancer through the lymph. Rectal cancer has two main transmission routes of a tumor by the lymph nodes. For the upper rectum, the tumor extends via the lymph nodes along the branches of the inferior mesenteric arteries. Lower portions of the rectum show the expansion of tumor in the lateral lymphatic flow, along the middle rectal vessels to the internal iliac arteries (26). Careful preoperative evaluation of the internal iliac area is significant for treating rectal cancer, especially in patients with carcinoma of the rectum in the lower portion, because the lymph nodes in that area lie outside the resection margin of the total mesorectal excision. Downward spread along the inferior rectal vessels to the groin is unusual except in very advanced cases, and also when the anal canal is involved (26).

Accuracy of CT and MRI scanning in nodal staging has been studied mostly for rectal cancer. Despite the high spatial resolution of the CT and MRI scans, which allows identification of lymph nodes as small as 2 to $3 \mathrm{~mm}$, reliable detection of nodal metastases is presently not possible. Radiological features, in assessing the involvement of lymph nodes, are mostly related to morphological criteria, such as the size and contour of the lymph nodes.

One meta-analysis showed 52\% sensitivity and $78 \%$ specificity of CT when it came to detecting nodal metastasis, and $65 \%$ sensitivity and $80 \%$ specificity for MRI scans. In another meta-analysis, the corresponding sensitivity and specificity were 55\% (95\% confidence interval: lower limit, 43; upper limit, 67) and 74\% (95\% confidence interval: 67.8 ) for CT and $66 \%$ (95\% confidence interval: 54.76 and $76 \%$ confidence interval: 59.87) for MRI scans (28).

The size of lymph nodes is a criterion which restricts analysis, since micro metastases in small lymph nodes are not recorded, and in large lymph nodes it is difficult to distinguish with certainty the presence of reactive hypertrophy from metastases. Rectal cancer is particularly known to have a high frequency of micrometastases in normal sized nodes $(13,30,32,33)$, with $45.4 \%$ to $78 \%$ of the involved nodes being $\leq 5 \mathrm{~mm}$ in size. Therefore, a lower size criteria in predicting malignant perirectal lymphadenopathy (such as $\geq 5 \mathrm{~mm}$ ), compared to the criteria for the other intra-abdominal nodal stations (such as $\geq 8 \mathrm{~mm}$ or $\geq 10 \mathrm{~mm}$ ), is recommended in the interpretation of perirectal lymph nodes. Morphological analysis of lymph nodes may help diagnose malignant lymph nodes to a certain degree. Speculated or blurred boundaries of lymph nodes, heterogeneous, mottled, and high signal intensity in the lymph node is characteristic of 
malignant lymphadenopathy on T2-weighted MRI imaging $(13,33)$.

\section{Diagnosis of distant metastases in rectal cancer}

Metastatic tumors are very common in the late stages of cancer. The spread of metastases may occur through the blood, the lymphatics, or through both routes. The most common places for metastasis to occur are the liver, lungs, brain and bones. The liver is the most common place of hematogenous dissemination of colorectal cancer with the emergence of a typical focal area of low density on the CT scan or high signal intensity on the T2WI in MRI scan, and low on T1WI, in comparison with normal liver parenchyma, with or without rim enhancement after contrast enhancement. The portal phase is the optimal time for scanning metastasis for lesions, and enables easy detection. The sensitivity of CT and MRI scans in detecting metastases is moderate. A CT scan is highly sensitive in detecting nods in the lungs, so it is preferred in diagnosing lung metastases in colorectal cancer, however it is not overly specific; also, the CT scan is sensitive in the detection of bone metastases. For brain metastasis MRI has been proven more suitable than a CT scan. A CT scan is limited in the diagnosis of the peritoneal spread of metastatic disease in colorectal carcinoma. Sensitivity per patient is $60 \%-76 \%$. Per lesion, sensitivity is limited, demonstrating only $9.1 \%$ to $24.3 \%$ for tumor implants smaller than $1 \mathrm{~cm}(9,34,35)$.

\section{Fluorodeoxyglucose-positron emission tomography (FDG-PET) in the preoperative diagnosis of rectal cancer}

There is controversy surrounding the routine preoperative staging of colorectal cancer with FDG-PET (36), and the role for FDG-PET in determining the stage of colorectal cancer has yet to be established (37). Preoperative FDG-PET imaging may be helpful in detecting distant metastases and could render surgery unnecessary in patients with increased surgical risk. It may be helpful as a baseline evaluation prior to neoadjuvant chemotherapy in patients with advanced stage disease (38).

Several meta-analyses have shown that FDG-PET was superior to CT or MRI in detection of hepatic metastases from various cancers of the gastrointestinal tract, with $90 \%$ sensitivity and $85 \%$ specificity, or similar to CT or MRI in the detection of hepatic metastases from colorectal cancer, with $75.9 \%$ sensitivity (39). This was demonstrated by a meta-analysis comparing non-invasive imaging methods (US, CT, MRI and FDG-PET) for the detection of hepatic metastases from colorectal cancer, gastric, and esophageal cancers, at an equivalent specificity of $85 \%$. FDG-PET had highest sensitivity (90\%) compared to MRI (76\%), CT (72\%), and US (65\%) (40). After an economic evaluation, as we see in the literature, we can conclude that FDGPET and Positron emission tomography CT (PET-CT), as an add-on imaging device, is cost-effective in the preoperative staging of recurrent colon, recurrent rectal and metastatic disease but not in primary colon or rectal cancer. According to the literature, it has low sensitivity in diagnosing FDGPET lymph nodes metastasis, and it is not recommended in routine diagnosis of metastatic colorectal carcinoma. In the study of Schmidt, et al. it was reported that accuracy for PET-CT was $91 \%$ (sensitivity $86 \%$, specificity 96 ) and $83 \%$ for MRI (sensitivity $72 \%$, and specificity 93\%) retrospectively. Initial results suggest that differences in accuracy for local and distant metastases detection, using FDG-PET and PET-CT and MRI for integrated screening of tumor recurrences in colorectal cancer, depend on the location 
of the malignant focus (41). FDG-PET can detect extra-hepatic metastases and determine whether to go for resection of hepatic metastases, if it would result in a longer benefit to the patient (39).

\section{Post-operative follow-up with the patients with rectal cancer}

Recurrences take place 3 to 5 years after treatment. An annual rigid proctoscopy, or barium enema, is used for monitoring patients after surgical treatment, together with radiological imaging methods CT and MRI scans. More than half the patients have postoperative relapse and distant metastases in the liver and lungs (42). A relapse is more likely to occur to the anastomosis as an extraluminal lesion, rather than intraluminal. Therefore, cross-section imaging, such as CT and MRI scans, plays an important role in the post-operative survival of patients after curative operation for colorectal cancer, because it primarily evaluates extra-colic structures. In addition to MSCT imaging, it is necessary to measure carcinoembryonic antigen (CEA) in the serum during the postoperative follow-up (43-45). The American Society of Clinical Oncology has revised the guide for colorectal cancer and suggests an annual abdominal CT control for 3 years after primary therapy, for patients who have a high risk of recurrence. A positive CT finding of local recurrence involves the TU mass with enlargement of the local lymph nodes, or invasion of surrounding structures, as well as ischiorectal fosses. In the diagnostics of pelvic recurrence, CT and MRI are used.

The diagnostic problem of scars and fibrosis after radiation therapy and local recurrence could not be resolved with classical CT scanning. Application of MRI scans is useful in assessing the extension of recurrence or post-operative scarring. Fibrosis has low signal intensity, as opposed to relapse in T2WI, which has high signal intensity. Some studies suggest that high signal intensity on T2WI can be found with a non-neoplastic inflammatory process, or edema and fresh fibrosis (less than 1 year old), while low signal intensity in a TU mass may occur in a recurrence with desmoplastic reaction.

\section{FGD-PET in post-operative follow-up for patients with rectal cancer}

Unlike CT and MRI scans, FDG-PET is highly sensitive in diagnosing suspected recurrence of colorectal cancer $(41,43)$. A number of studies have demonstrated the role of FDG-PET as a metabolic imaging modality for detecting recurrent or metastatic disease. The sensitivity of FDG-PET is in the $90 \%$ range, with specificity greater than $70 \%$, both superior to CT scans. A meta-analysis of 11 clinical reports and 577 patients showed that the sensitivity and specificity of FDG-PET for detecting recurrent colorectal carcinoma was $97 \%$ and $76 \%$ respectively (46). False negative FDG-PET findings have been reported with mucinous adenocarcinoma (47). It is applied in distinguishing a recurrence from postoperative fibrosis in the pelvic area. The residue or recurrence tumor shows accumulation of the radio-tracer (47). It is recommended for patients with elevated CEA, without the presence of radiological signs of recurrence or metastasis (9).

\section{New guidelines for colorectal carcinoma using modern diagnostic imaging in monitoring patients with a tumor}

Today, international standards related to the size of tumors are significant but insufficient in indicating the effects of therapy on the biology of the tumor, as well as indicating the success rate of the applied therapy. Modern diagnostic imaging, such as perfused ultrasound imaging, perfused CT or MRI scans, 
diffusion MRI, and metabolic imaging with FDG-PET and PET-CT, has an important role in monitoring patients with a tumor (37). In the future, a range of these highly efficient imaging techniques will be applied not only as diagnostic imaging in the early diagnosis of cancer, but also as a routine diagnostic method in each oncology department for chemo or radio therapy. Which methods will be used depends on the CEA in the serum during post operative followup. It is recommended that for patients with elevated CEA, without radiological signs of recurrence or metastasis present, metabolic imaging (PET-CT) should be used, which has an important role in monitoring patients with a tumor (43).

\section{Conclusion}

In recent years with the improvement of cross-section imaging, the widespread use of MSCT scans, as well as the technologically improved MRI units of 1.5 and 3T, the diagnosis of primary rectal cancer and recurrences, or metastases occurrence in postoperative patients with elevated CEA, is safer. Patients are more likely to survive rectal cancer by following the proposed guidelines for diagnosing and post-operative monitoring. MSCT imaging has a primary role in diagnosing and evaluating the process, and monitoring, disease prognosis and monitoring of possible complications. For rectal cancer, the application of TRUS and MRI scanning in diagnosis and differentiating post-operative and post-irradiation fibrosis in relation to relapse, is an advantage compared to MSCT imaging. Today, in the pre-operative stages, rectal MRI becomes mandatory.

Conflict of interest: The authors declare that they have no conflict of interest. This study was not sponsored by any external organization.

Authors' contributions: Conception and design: LLC and $\mathrm{MBC}$; Acquisition, analysis and interpretation of data: DV and SVZ; Drafting the article: LLC and MBČ; Revising it critically for important intellectual content: LLC and MBČ.

\section{References}

1. Jemal A, Siegel R, Ward E, Hao Y, Xu J, Thun MJ. Cancer statistics, 2009. CA Cancer J Clin. 2009;59:225-9.

2. Winawer SJ, Stewart ET, Zauber AG, Bond JH, Ansel H, Waye JD, et al. A comparison of colonoscopy and double-contrast barium enema for surveillance after polypectomy. National polyp Study Work Group. N Engl J Med. 2000;342:1766-72.

3. McFarland EG, Levin B, Lieberman DA, Pickhardt PJ, Johnson CD, Glick SN, et al. Revised colorectal screening guidelines: Join effort of the American Cancer Society, U.S. Multisocety Task Force on Colorectal Cancer, and American College of Radiology. Radiology. 2008;248:717-20.

4. Punwani S, Halligan S, Tolan D, Taylor SA, Hawkes D. Quantitative assessment of colonic movement between prone and supine patient positions during CT colonography. British Journal of Radiology. 2009;82:475-81.

5. Mehdy Chadi. CT Colonography (virtual colonoscopy). Guerbet. 2005;12:5.

6. Zalis ME, Barish MR, Choi JR, Dachman AH, Fenolou HM, Ferrucci JT, et al. For the working group on Virtual Colonoscopy. CT Colonoscopy Reporting and Data System: A Consensus Proposal. Radiology. 2005;236:3-9.

7. Balchar A, Suosa J. CT colonography (virtual colonoscopy): technique indications and performance. Digestion. 2007;76:34-41.

8. Sofić A, Bešlić Š, Kocijanić I, Šehović N. CT colonography in detection of the colorectal carcinomas. Radiol Oncol. 2008;42(3):136-42.

9. Gollub MJ, Schwartz LH, Akhurst T. Up date on colorectal cancer imaging. Radiol Clin North Am. 2007;45:85-118.

10. Roddie M. CT colonography tools advance in clinical use. Diagnostic Imaging Europe. 2006;10:35-7.

11. Liedenbaum MH, de Vries AH, Gouw CI, van Rijn AF, Bipat S, Dekker E, Stoker J. CT colonography with minimal bowel preparation; evaluation of tagging quality, patient acceptance and diagnostic accuracy in two iodine-based preparation schemes. Eur Radiol. 2010;20:367-76.

12. Luz O, Buchgeister M, Klabunche M, Trabold T, Kopp AF, Claussen CD, et al. Evaluation of dose exposure in 64-slice CT colonography. Eur Radiol. 2007;17:2616-21. 
13. Brown G, Daniels IR, Richardson C, Revell P, Peppercorn $\mathrm{D}$, Bourne $\mathrm{M}$. Techniques and trouble shooting in high spatial resolution thin slice MRI for rectal cancer. Br J Radiol. 2005;78:245-51.

14. Salermo G, Daniels IR, Brown G. Magnetic resonance imaging of low rectum. Defining the radiological anatomy. Colorectal Dis. 2006;8(Supp 3):10-3.

15. Edge SB, Byrd DR, Compton CC, Fritz AG, Greene FL, Trotti A, editors. AJCC Cancer Staging Manual, 7th ed. New York (etc.): Springer; 2010 [cited 2012 May 31]. Available from: http://www. cancerstaging.org/staging/index.html

16. Iafrate F, Laghi A, Paolantonio P, Rengo M, Mercantini P, Ferri M, et al. Preoperative staging of rectal carcinoma with MRI imaging correlation with surgical and histopathologic findings. Radiographics. 2006;26:701-14.

17. Kim NK, Kim MJ, Park JK, Park SI, Min JS. Preoperative staging of rectal cancer with MRI: accuracy and clinical usefulness. Ann Surg Oncol. 2000;7:732-7.

18. Burt S, Brown G, Daniels IR, Norman AR, Mason B, Cunningham D. MRI directed multidisciplinary team preoperative treatment strategy: the way to eliminate positive circumferential margins. Br J Cancer. 2006;94(3):351-7.

19. Maier AG, Kersting-Sommerhoff B, Reeders JW, Judmaier W, Schima W, Annweiller AA, et al. Staging of rectal cancer by double-contrast MR imaging using the rectally administered super paramagnetic iron oxide contrast agent ferristene and IV gadodiamid injection: results of a multicenter phase II trial. J Magn Reson Imaging. 2000; 12:657-60.

20. Klessen C, Rogalla P, Taupitz M. Local staging of rectal cancer: the current role of MRI. Eur Radiol. 2007;17:379-89.

21. Berman L, Israel GM, McCarthy SM, Weinreb JC, Longo WE. Utility of the magnetic resonance imaging in anorectal disease. World J Gastroenterol. 2007;13:3153-8.

22. Sofić A, Šehović N, Prnjavorac B, Bilalović N, Čaluk J, Sofić D. MR rectum imaging with ultrasound gel as instrumental contrast media in tubulovillous adenoma. Radiol Oncol. 2008;42(3):136-42.

23. Beets-Tan RG, Beets GL, Vliegen RF, Kessels AG, Van Boven H, De Bruine A, et al. Accuracy of magnetic resonance imaging in predicting of tumor-free resection margin in rectal cancer surgery. Lancet. 2001;375:497-504.

24. Wibe A, Rendedal PR, Svensson E, Norstein J, Eide TJ, Myrvold HE, et al. Prognostic significance of the circumferential resection margin fol- lowing total mesorectal excision for rectal cancer. Br J Surg. 2002;89:327-34.

25. Taylor SA, Halligan S, Goh V, Morley S, Bassett P, Atkin W, et al. Optimizing colonic distention for multi-detector row CT colonography: effect of hyoscine butylbromide and rectal balloon catheter. Radiology. 2003;229(1):99-108.

26. Beets-Tan RG, Beets GL. Rectal cancer: review with emphasis on MR imaging. Radiology. 2004;232:335-46.

27. Kwok H, Bissett IP, Hill GL. Preoperative staging of rectal cancer. Int J Colorectal Dis. 2000;15:9-20.

28. Bipat S, Glas AS, Slors FJ, Zwinderman AH, Bossuyt PM, Stoker J. Rectal cancer: local staging and assessment of the lymph node involvement with endoluminal US, CT and MR imaging - a meta analysis. Radiology. 2004;232:773-83.

29. Geeneu RW, Hussain SM, Cademartiri F, Poley JW, Siersema PD, Krestin GP. CT and MR colonography: scanning techniques, post-processing and emphasis on polyp detection. Radiographics. 2004;24:e18.

30. Martling A, Holm T, Bremmer S, Lindholm J, Cedermark B, Blomqvist L. Prognostic value of preoperative magnetic resonance imaging of the pelvis in rectal cancer. Br J Surg. 2003;90(1):1422-8.

31. O’Neill BD, Salerno G, Thomas K, Tait DM, Brown G. MR vs CT imaging: low rectal cancer tumour delineation for three-dimensional conformal radiotherapy. Br J Radiol 2009;82:509-13.

32. Mönig SP, Baldus SE, Zirbes TK, Schröder W, Lindemann DG, Dienes HP, et al. Lymph node size and metastatic infiltration in colon cancer. Ann Surg Oncol. 1999;6:579-81.

33. Kim JH, Beets GL, Kim MJ, Kessels AG, Beets-Tan RG. High- resolution MR imaging for nodal staging in rectal cancer: Are there any criteria in addition to the size? Eur J Radiol. 2004;52:78-83.

34. de Bree E, Koops W, Kröger R, van Ruth S, Witkamp AJ, Zoetmulder FA. Peritoneal carcinomatosis from colorectal or appendicle origin: Correlation of preoperative CT with intraoperative findings and evaluation of interobserver agreement. J Surg Oncol. 2004;86:64-73.

35. Heunedige T, Teo L, Ang B, Cheong WK, Venkatesh SK. Accuracy of preoperative CT for local staging in colorectal carcinomas. Singapore Med J. 2010;51(6):457-80.

36. Furukawa H, Ikuma H, Seki A, Yokoe K, Yuen S, Aramaki T, et al. Positron emission tomography scanning is not superior to whole body multidetector helical computed tomography in preoperative staging of colorectal cancer. Gut. 2006;55:1007-11. 
37. Long Sun, Hua Wu, Yong-Song Guan. Colonography by $\mathrm{CT}$, MRI and PET CT combined with conventional colonoscopy in colorectal cancer screening and staging. World J Gastroenterology. 2008;14(6):858-63.

38. Kiner S, Anatoch G, Bokisch A, Veit-Haibach P. Whole - body PET/CT colonography: a possible new concept - for coloreclal cancer staging. Abdom Imaging. 2007;32:606-12.

39. Bipat S, van Leeuwen MS, Comans EF, Pijl ME, Bossuyt PM, Zwinderman AH, et al. Colorectal liver metastases: CT, MR imaging, and PET for diagnosis - meta analisys. Radiology. 2005;237:123-31.

40. Kinkel K, Lu Y, Both M, Waren RS, Thoeni RF. Detection of hepatic metastases from cancers of the gastrointestinal tract by using noninvasive imaging methods (US, CT, MR imaging,PET): A meta analysis. Radiology. 2005;224:748-56.

41. Schmidt GP, Bauer-Melnyk A, Hang A, Utzschneider S, Beeker CR, Tiling R, et al. Whole - body MRI at 1.5 and 3 T compared with FDG-PET-CT for detection of tumor recurrence in patients with colorectal cancer. Eur Radiol. 2009;19:1366-78.

42. Manfredi S, Bouvier AM, Lepage C, Hatem C, Dancourt V, Faivre J. Incidence and patterns of recur- rence after resection for cure of colon cancer in a well defined population. Br J Surg. 2006;93:1115-22.

43. Flamen P, Hoekstra OS, Homans F, Van Cutsem E, Maes A, Stroobants S, et al. Unexplained rising carcinoembryonic (CEA) in the postoperative surveillance of colorectal cancer. The utility of positron emission tomography (PET). Eur J Cancer. 2001;31:862-9.

44. Marcus J, Morrissey B, deGara C, Tarulli G. MRI of recurrent rectosigmoid carcinomas . Abdom Imaging. 1997;22:338-42.

45. Schaefer O, Langer M. Detection of recurrent rectal cancer with CT, MRI and PET/CT. Eur Radiol. 2007;17:2044-54.

46. Huebner RH, Park KC, Shepherd JE, Schwimmer J, Czernin J, Phelps ME, et al. A meta-analysis of the literature for whole-body FDGPET detection of colorectal cancer. J Nucl Med. 2000;41:1177-89.

47. Whiteford MH, Whiteford HM, Yee LF, Ogunbioyi OA, Dehdashti F, Siegel BA, et al. Usefulness of FDGPET scan in the assessment of suspected metastatic or recurrent adenocarcinoma of the colon and rectum. Dis Colon Rectum. 2000;43:759-67. 